\title{
Proteoglycan depletion and size reduction in lesions of early grade chondromalacia of the patella
}

Urho Väätäinen, Tomi Häkkinen, Ilkka Kiviranta, Heikki Jaroma, Ritva Inkinen, Markku Tammi

\begin{abstract}
Objective-To determine the content and molecular size of proteoglycans (PGs) in patellar chondromalacia (CM) and control cartilages as a first step in investigating the role of matrix alterations in the pathogenesis of this disease.

Methods-Chondromalacia tissue from 10 patients was removed with a surgical knife. Using identical techniques, apparently healthy cartilage of the same site was obtained from 10 age matched cadavers (mean age 31 years in both groups). Additional pathological cartilage was collected from 67 patients with grades II-IV CM (classified according to Outerbridge) using a motorised shaver under arthroscopic control. The shaved cartilage chips were collected with a dense net from the irrigation fluid of the shaver. The content of tissue PGs was determined by Safranin $O$ precipitation or uronic acid content, and the molecular size by mobility on agarose gel electrophoresis.
\end{abstract}

Results-The mean PG content of the CM tissue sampled with a knife was dramatically reduced, being only $15 \%$ of that in controls. The cartilage chips collected from shaving operations of grades II, III, and IV CM showed a decreasing PG content: $9 \%, 5 \%$, and $1 \%$ of controls, respectively. Electrophoretic analysis of PGs extracted with guanidium chloride from the shaved tissue samples suggested a significantly reduced size of aggrecans in the mild (grade II) lesions.

Conclusion-These data show that there is already a dramatic and progressive depletion of PGs in CM grade II lesions. This explains the softening of cartilage, a typical finding in the arthroscopic examination of CM. The PG size reduction observed in grade II implicates proteolytic attack as a factor in the pathogenesis of $\mathrm{CM}$.

(Ann Rheum Dis 1995; 54: 831-835)

Softening of articular cartilage in the patella, frequently described as chondropathy or chondromalacia of the patella, varies from a soft blister to total destruction of the cartilage. Although chondromalacia of the patella is a common phenomenon, its aetiology is unclear; mechanical factors have been considered important, however. ${ }^{1}$
The matrix of cartilage consists of a collagenous network and interfibrillar aggregates formed by proteoglycans (PGs) with hyaluronan. PGs are extremely hydrophilic and adsorb water into the compartment ramified by the collagenous network. As a consequence of water absorption, the matrix remains turgid, creating the resilient nature of the cartilage. ${ }^{2-4}$ PGs are therefore essential for the compressive stiffness of cartilage matrix, while the collagen fibres are responsible for the tensile strength of articular cartilage. ${ }^{3}$

The chondrocytes synthesise and secrete PGs into the matrix and, in conditions of normal turnover, degraded PGs are released from articular cartilage into synovial fluid..$^{5-7}$ In experimental osteoarthritis, the total content of PGs decreases despite their increased biosynthesis, indicating an abnormally rapid loss of PGs. ${ }^{8-12}$ Focal loss of PGs has been found in canine patellar cartilage after acute transarticular patellofemoral loading. ${ }^{13}$ Reduced concentrations of PG also occur in the articular cartilage after immobilisation of the canine knee. ${ }^{14} 15$ With aging and often in osteoarthritis, the electrophoretic mobility of PG monomers is increased, indicative of a reduction in their molecular size. ${ }^{16}{ }^{17}$

However, little is known about the PGs in chondromalacia of the patella, though it is the most frequently diagnosed cartilage lesion in the knee joint. The purpose of the present study was to examine the PG content of chondromalacia lesions of different grades, and to begin to characterise the structural alterations of PGs in the diseased matrix.

\section{Materials and methods}

The study was approved by the ethics committee of the Kuopio University Hospital and all the subjects gave their informed consent to take part in the study.

CARTILAGE SAMPLES

Patients with anterior knee pain were examined clinically and knee joint roentgenograms were taken. Those patients giving a strong suspicion of a patellofemoral problem, but without radiological signs of osteoarthritis, were recruited to the study. Athroscopy of the knee was performed in 130 patients, and 77 showed chondromalacia of the patella (CM) as the only intra-articular cartilage lesion. At operation, the fibrillated cartilage of the CM lesion was 
shaved smooth to reduce pain, and the shaved tissue taken for PG analysis. Sixty seven of these cartilage samples were taken arthroscopically, and 10 were taken during open arthrotomy with a knife. At arthroscopy, CM lesions were classified into four grades according to Outerbridge. ${ }^{18} \mathrm{We}$ recruited to the study only those patients with chondromalacia of the patella who showed intact cartilage surfaces in the other areas of the knee joint.

Cartilage tissue from CM lesions of 10 patients (six women, four men; mean age 31 years, range 21-45) was removed in as intact a condition as possible, using a surgical knife and cutting perpendicularly to the articular surface down to the subchondral bone, which was left intact. Open surgery in these patients was indicated for lateral release in four patients, patellar realignment in four, and periosteal transplantation in two patients. Using identical techniques, apparently healthy cartilage from the same sites was obtained within 24 hours after death from 10 age matched cadavers (mean age 31 years, range 19-42). Cadavers with known autoimmune diseases, haemophilia, and metabolic disorders were excluded. Additional pathological cartilage was collected from 67 patients (mean age 35.0 (SD 9.9) years) with grades II-IV CM using a motorised shaver (Stryker, Stryker Endoscopy, Sunnyvale, USA) with an aggressive meniscus cutter blade of $5 \mathrm{~mm}$ diameter under arthroscopic control. Forty eight percent of the chondromalacia lesions were located on medial facet of the patella, $39 \%$ were on the central area (diameter of $15 \mathrm{~mm}$ ), and $13 \%$ were located on the lateral facet of the patella. As the cartilage lesion was smoothed, the shaved cartilage tissue was collected with a dense cotton net from the irrigation solution $(0.9 \%$ sodium chloride) leaving the joint. The samples were put into one millilitre Eppendorf tubes and frozen at $-42^{\circ} \mathrm{C}$.

Bovine knees were obtained from the local abattoir and sampled with a shaver and knife using exactly the same technique as for the human cartilages.

\section{HISTOLOGY}

Cartilage samples were taken for histology from $10 \mathrm{CM}$ samples and from 10 cadaver control samples. The tissue was fixed in buffered formaldehyde solution, dehydrated through an alcohol series to xylene and embedded in paraffin. Sections $3 \mu \mathrm{m}$ thick were cut and stained with haematoxylin-eosin.

\section{EXTRACTION OF PROTEOGLYCANS}

The wet cartilage samples were weighed. Those from cadavers and those taken from patients with a knife were sliced with a microtome to a thickness of $20 \mu \mathrm{m}$ at $-20^{\circ} \mathrm{C}$, and lyophilised. Cartilage samples obtained from shaving operations in 26 patients in the CM group (mean age 35.0 (SD 9.0 ) years) were extracted without further cutting.

PGs were extracted from cartilage samples of $71.9-406.9 \mathrm{mg}$ wet weight with $1.5 \mathrm{ml}$ of
$4 \mathrm{~mol} / \mathrm{l}$ guanidinium chloride ( $\mathrm{GuCl}$, Fluka, Buchs, Switzerland) in $50 \mathrm{mmol} / \mathrm{l}$ sodium acetate, $\mathrm{pH} 5.8$ at $4^{\circ} \mathrm{C}$. Disodium-EDTA $10 \mathrm{mmol} / 1$ (Sigma Chemical Co, St Louis, MO, USA), $100 \mathrm{mmol} / 1 \mathrm{\epsilon}$-amino-n-caproic acid, $5 \mathrm{mmol} / 1$ benzamidine hydrochloride (Sigma), and $0.02 \%$ (weight/volume) sodium azide (Merck, Darmstadt, Germany), were used as protease and bacterial growth inhibitors.

After extraction, all the samples were transferred into deionised water through a desalting column (PD-10, Pharmacia, Sweden). PGs were quantitated by Safranin O precipitation ${ }^{19}$ using chondroitin sulphate $\mathrm{C}$ (Sigma) as a standard, or by uronic acid determination. ${ }^{20}$

\section{AGAROSE GEL ELECTROPHORESIS}

Each sample was analysed by electrophoresis in horizontal, submerged $3 \mathrm{~mm}$ thick $0.75 \%$ (w/v) agarose slab gels as described previously. ${ }^{21}$ Relative mobilities were compared with a chondroitin sulphate standard (shark cartilage, Sigma), and with bovine articular cartilage A1 PG, electrophoresed in the same gel.

The gels were stained with toluidine blue, dried on a Gel-Bond film (FMC BioProducts, Rockland, USA) and scanned with a tabletop grey scale scanner (256 shades of grey) connected to a microcomputer (Macintosh II, Apple, Cupertino, Ca, USA). The scanned images of the gels were analysed for stain density and graphically averaged by image analysis software (Image, public domain software by W Rasband, NIH, USA).

\section{STATISTICAL METHODS}

Mann-Whitney $U$ test was applied to test the differences between control and chondromalacia groups. To examine correlations, Pearson's squared correlation test was used.

\section{Results}

Knee joint cartilage surfaces were carefully studied to exclude as far as possible any variance associated with joint diseases other than chondromalacia of the patella. If other cartilage lesions were observed at arthroscopy, the samples were not accepted for the study. Patients with irritated plicas were excluded also.

Control cartilages showed a normal histological appearance (fig 1A). Cartilage changes in chondromalacia included fibrillation of the joint surface and erosion of the cartilage (fig 1B-D).

\section{PROTEOGLYCAN CONTENT IN} CHONDROMALACIA

The concentration of uronic acid in chondromalacia cartilage was decreased on average to $15 \%$ of that in controls (table 1). A larger number of samples from shaving operations were used to estimate the relationship between PG concentration and grade of lesion. The 


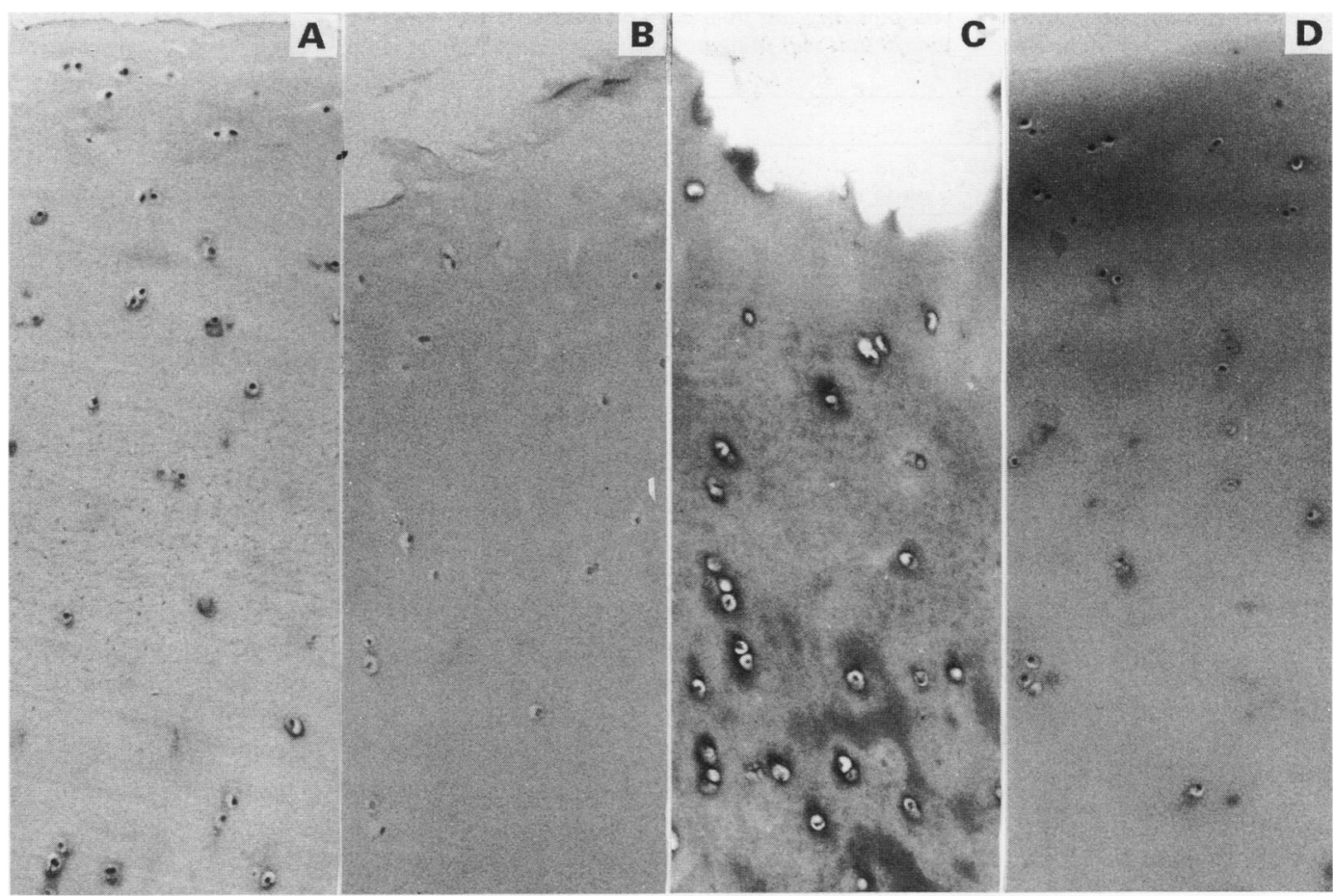

Figure 1 Haematoxylin and eosin stained histological sections. A: Control cartilage showing normal feature of cartilage structure; B: grade II chondromalacia-superficial zone partly lost; C, D: grades III and IV-superficial zone and part of the intermediate zone have been eroded. Original magnification $\times 10$.

decrease in the concentration of uronic acid was greatest in the most advanced lesions (grade IV), but no statistically significant differences between the grades were apparent (table 2).

The overall concentration of uronic acid was smaller in the samples harvested from shavings, compared with those taken with the knife, apparently as a result of swelling of the shaved tissue chips and possibly, also, some extraction of the PGs in the irrigation fluid.

\section{AGAROSE GEL ELECTROPHORESIS OF}

PROTEOGLYCANS

Figure 2 shows the graphically averaged PG profiles of control and chondromalacia samples (grades II-IV). The broad peak in all groups corresponds to aggrecan, which is very heterogeneous in human articular cartilage. ${ }^{22-24}$ The smaller PGs (decorin and biglycan) migrate at

Table 1 Uronic acid concentration in the patellar cartilage of patients with chondromalacia (CM) of the patella, and in apparently normal, age matched cadaver controls

\begin{tabular}{llll}
\hline Group & $\begin{array}{l}\text { Sex } \\
(F / M)\end{array}$ & $\begin{array}{l}\text { Uronic acid } \\
(\mu g / m g \text { wet wt) }\end{array}$ & $\begin{array}{l}\text { \% of } \\
\text { control }\end{array}$ \\
\hline Controls $(\mathrm{n}=10)$ & $1 / 9$ & $3.61(1.78)$ & 100 \\
$\mathrm{CM}(\mathrm{n}=10)$ & $6 / 4$ & $0.53(0.62)^{\star \star \star}$ & 15 \\
\hline
\end{tabular}

Values are mean (SD).

$\star \star \star \mathrm{p}<0.001$, Mann-Whitney $U$ test.

Table 2 Uronic acid concentration (mean (SD)) in different grades of chondromalacia (CM) of the patella, measured in samples harvested from the irrigation fluid during shaving of cartilage lesions

\begin{tabular}{lll}
\hline $\begin{array}{l}\text { Grade of } \\
C M\end{array}$ & $\begin{array}{l}\text { Sex } \\
(F / M)\end{array}$ & $\begin{array}{l}\text { Uronic acid } \\
(\mu g / m g \text { wet wt })\end{array}$ \\
\hline II & $13 / 12$ & $0.33(1 \cdot 06)$ \\
III & $14 / 16$ & $0 \cdot 12(0 \cdot 14)$ \\
IV & $10 / 2$ & $0.05(0 \cdot 04)$ \\
\hline
\end{tabular}
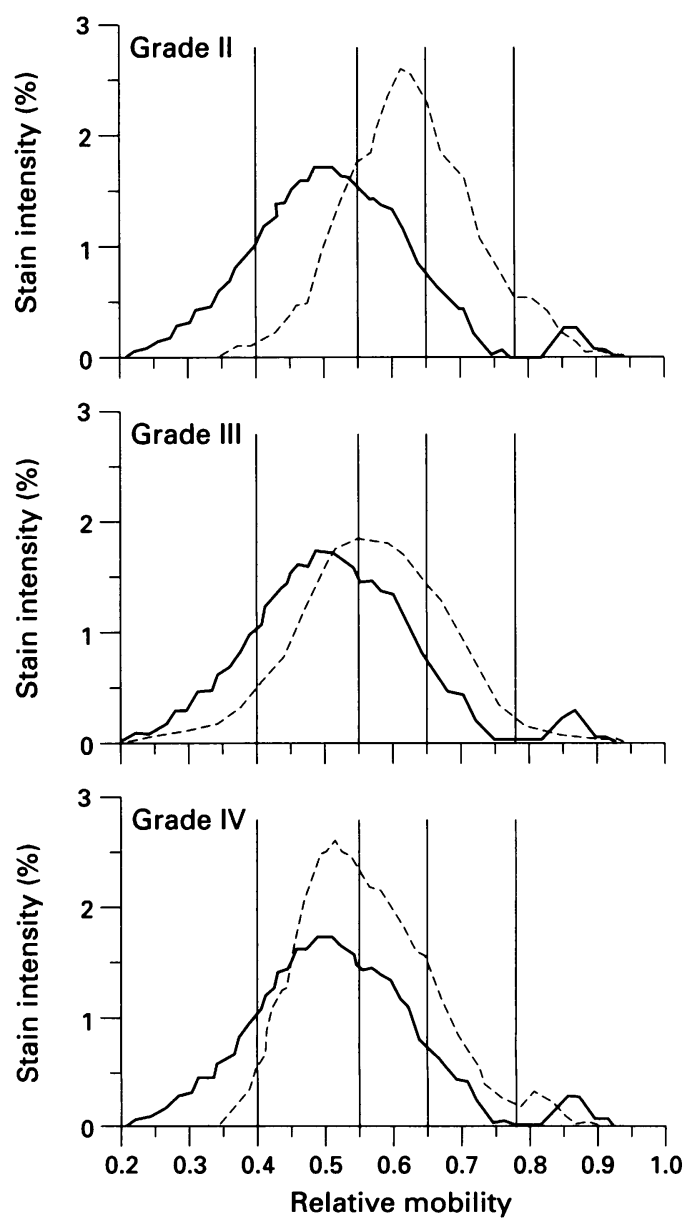

Figure 2 Agarose gel electrophoresis of cartilage

proteoglycans from grades II-IV of chondromalacia patellae. $(--)$ and controls (-). Densitograms of toluidine blue stained gels, expressed as percentage of total stain intensity in a sample. Each line represents a graphic average of individual samples. Vertical lines define five segments for purposes of comparing mobilities (see text and table 3). 
Table 3 Relative mobility $\left(\mathrm{R}_{F}\right)$ of the proteoglycans from different grades (CMII-CMIV) of chondromalacia of the patella and controls $(C)$ in agarose gel electrophoresis: five profile segment as shown in figure 1

\begin{tabular}{lcllll}
\hline Group & \multicolumn{3}{l}{ Mobility $\left(\mathrm{R}_{F}\right)$} & & \\
\cline { 2 - 6 } & $\leqslant 0.40$ & $\begin{array}{l}>0.40 \\
\leqslant 0.55\end{array}$ & $\begin{array}{l}>0.55 \\
\leqslant 0.65\end{array}$ & $\begin{array}{l}>0.65 \\
\geqslant 0.78\end{array}$ & $>0.78$ \\
\hline C (n=7) & $15(17)$ & $46(15)$ & $26(11)$ & $9(11)$ & $3(6)$ \\
CMII (n=6) & $1(1)^{\star}$ & $20(13)^{\star}$ & $40(6)^{\star}$ & $32(11)^{\star \star}$ & $7(6)$ \\
CMIII (n=16) & $5(8)$ & $35(17)$ & $33(9)$ & $22(13)^{\star}$ & $4(7)$ \\
CMIV (n=4) & $3(2)$ & $48(8)$ & $31(6)$ & $15(2)$ & $3(6)$ \\
\hline
\end{tabular}

Values are percentage (SD)

${ }^{\star} \mathrm{p}<0.05,{ }^{\star \star} \mathrm{p}<0.01$, compared with controls (Mann-Whitney $U$ test).

$R_{\mathrm{F}} 0.8-0.9$ in the electrophoretic system used. To allow quantitative comparison of PG mobilities between control and chondromalacia samples, the profiles were split into the five segments shown in figure 2 . The integrated stain intensity in each segment was calculated to provide the values shown in table 3 . The data show a significant decrease in the low mobility segments $\left(R_{\mathrm{F}}<0.55\right)$ in grade II chondromalacia, and a corresponding significant increase in the high mobility segments $\left(R_{\mathrm{F}}\right.$ $0.55-0.78)$. In grade III chondromalacia, the high mobility segment $\left(R_{\mathrm{F}} 0 \cdot 65-0.78\right)$ was significantly increased (table 3 ). Statistically significant differences of PG mobilities between controls and grade IV chondromalacia were not observed.

EFFECT OF SHAVING ON PROTEOGLYCAN SIZE Electrophoretic analysis of paired cartilage samples obtained from bovine knee articular cartilage with a knife and shaver revealed no difference in PG size, thus excluding any artificial PG degradation arising from the shaving procedure.

\section{Discussion}

These data have demonstrated a severe and progressive depletion of PGs in chondromalacia of the patella grade II-IV lesions. This explains the typical finding of a softening of cartilage in arthroscopic examination of CM. The smaller size of PGs extracted from the mild lesions may reflect proteolysis as a factor in the initiation of the lesion.

The importance of a sufficient content of PG and an intact collagen network to the normal stiffness of articular cartilage has been reported in several studies. ${ }^{25-27}$ Our results support these findings, and indicate that the softening regularly noted in chondromalacia is related to disappearance of PGs from the tissue. Softening of articular cartilage is a dominant feature even of grade I chondromalacia with an intact cartilage surface, indicating that PG loss is an early phenomenon in the degenerative process.

The association between cartilage softening and PG loss can be used to detect early changes in cartilage matrix by measuring its stiffness (elastic modulus) with a novel indentation device applicable during arthroscopy. ${ }^{28} 29$ This should provide quantitative follow up measurements on the condition of the cartilage matrix even in grade I cases.
The turnover of cartilage matrix involves the proteolytic cleavage of the core protein of aggrecan, the predominant cartilage PG (on a mass basis). ${ }^{30}$ Uncontrolled proteolysis may well account for the PG loss in chondromalacia. The present results demonstrated a significant size reduction in the large PGs which is in agreement with the concept that, at least in the initial stages of the disease, the tissue and its aggrecans have been subject to severe attack by proteinase(s). ${ }^{31}$ However, until metabolic data are available, it is possible that long term inhibition of aggrecan synthesis may explain the greatly reduced quantities of apparently degraded aggrecans present in the chondromalacia patellae lesions.

The absence of size reduction of PGs in severe (grade IV), compared with early, chondromalacia may reflect a complete loss of the PGs originally present in the tissue and their replacement by molecules newly synthesised through activated repair, or molecules from the healthy rim of the lesion. This concept is supported by observations in osteoarthritic cartilage, which show degradation of PGs in the initial phase, followed later by replacement of PGs with larger aggregating molecules. ${ }^{31}$ In addition, large aggrecans are present particularly in new, osteophytic cartilage. ${ }^{31}$

Our findings clearly establish the major role of the PG matrix in chondromalacia of the patellae, and warrant further studies on factors leading to the PG depletion.

This study was supported by grants from the North Savo Regional Fund of the Finnish Cultural Foundation and the Foundation of Finnish Orthopaedics and Traumatology.

1 Insall $\mathrm{J}$, Falvo $\mathrm{K} \mathrm{A}$, Wise $\mathrm{D} \mathrm{W}$. Chondromalacia patellae. f Bone foint Surg Am 1976; 1: 1-8.

2 Hardingham T E, Fosang A J, Dudhia J. Aggrecan, the chondroitin sulfate/keratan sulfate proteoglycan from Hascall V C, eds. Articular cartilage and osteoarthritis. New York: Raven Press, 1992; 5-20.

3 Mayne R, Irwin M H. Collagen types in cartilage. In: Kuettner K E, Schleyerbach R, Hascall V C, eds. Articular Kuettner K E, Schleyerbach R, Hascall V C, eds. Articular
cartilage biochemistry. New York: Raven Press, 1986; cartilage

4 Kuettner $\mathrm{K}$. Biochemistry of articular cartilage in health and disease. Clin Biochem 1992; 25: 155-63.

5 Witter J, Roughley P, Webber C, Roberts N, Keystone E, Poole A R. The immunologic detection and characterization of cartilage proteoglycan degradation products in synovial fluids of patients with arthritis. Arthritis Rheum 1987; 30: 519-29.

6 Carrol G J. Spectrophotometric measurement of proteoglycans in osteoarthrotic synovial fluid. Ann Rheum Dis 1987; 46: 375-9.

7 Tyler J A. Chondrocyte-mediated depletion of articular cartilage proteoglycans in vitro. Biochem $f$ 1985; 225: 493-507.

8 Bayliss M T Metabolism of animal and human osteoarthritic cartilage. In: Kuettner K E, Schleyerbach R, Peyron J G, Hascall V C, eds. Articular cartilage and osteoarthritis. New York: Raven Press, 1992; 487-500.

9 Carney S L, Billingham M E J, Muir H, Sandy J D. Structure of newly synthesized proteoglycans and proteoglycan turnover products of cartilage explant culture from dogs with experimental osteoarthritis. fOrthop Res 1985; 3: 140-7.

10 Carney S L, Billingham M E J, Muir H, Sandy J D. Demonstration of increased proteoglycan turnover in cartilage explants from dogs with experimental osteoarthritis. F Orthop Res 1987; 2: 201-6.

11 Carney S L, Billingham M E J, Caterson B, et al. Changes in proteoglycan turnover in experimental canine osteoarthritic cartilage. Matrix 1992; 12: 137-47.

12 Lafeber F P J G, van der Kraan P M, van Roy H L A M, et al. Local changes in proteoglycan synthesis during culture are different for normal and Am $\mathcal{f}$ Pathol 1992; 6: 1421-9.

13 Thompson R C, Vener M J, Griffiths H J, Lewis J L, Oegema T R, Wallace L. Scanning electron-microscopic and magnetic resonance-imaging studies of injuries to the 
patellofemoral joint after acute transarticular loading. f Bone foint Surg Am 1993; 5: 704-13.

14 Palmoski M J, Perricone E, Brandt K D. Development and reversal of a proteoglycan aggregation defect in normal reversal of a proteoglycan aggregation defect in normal Canine knee cartilage 17

15 Kiviranta I, Jurvelin J, Tammi M, Säämänen A-M, Helminen $\mathrm{H} \mathrm{J}$. Weight-bearing controls glycosaminoglycan concentration and articular cartilage thickness in the knee joints of young Beagle dogs. Arthritis Rheum 1987; 30: 801-9.

16 Rosenberg L C, Buckwalter J A. Cartilage Proteoglycans. In: Kuettner K E, Schleyerbach R, Hascall V C, eds. Articular cartilage biochemistry. New York: Raven Press, 1986; 39-57.

17 Witsch-Prehm P, Miehlke R, Kresse H. Presence of small proteoglycan fragments in normal and arthritic human proteoglycan fragments in normal and arthe. Arthritis Rheum 1992; 9: 1042-52.

18 Outerbridge R E. The etiology of chondromalacia patellae. f Bone foint Surg Br 1961; 4: 752-7.

19 Lammi M, Tammi M. Densitometric assay of nanogram quantities of proteoglycans precipitated on nitrocellulose membrane with Safranin O. Anal Biochem 1988; 168: 352-7.

20 Blumenkranz N, Asboe-Hansen G. New method for quantitative determination of uronic acids. Anal Biochem 1973; 54: 484-9.

21 Säämänen A-M, Tammi $M$, Kiviranta I, Jurvelin J, Helminen $\mathrm{H}$ J. Levels of chondroitin-6-sulfate and nonaggregating proteoglycans at articular cartilage contact sites in the knees of young dogs subjected to moderate running exercise. Arthritis Rheum 1989; 32: 1289-93.
22 Bayliss $M T$. Proteoglycan structure in normal and osteoarthrotic human cartilage. In: Kuettner $\mathrm{K} \mathrm{E}$, Schleyerbach R, Hascall V C, eds. Articular cartilage biochemistry. New York: Raven Press, 1986; 295-310.

23 Nietfeld J J. Cytokines and proteoglycans. Experientia 1993; 49: 456-69.

24 Yanagishita $M$. Function of proteoglycans in the extracellular matrix. Acta Pathol fpn 1993; 43: 283-93.

25 Kempson G E. Mechanical properties of human cartilage [Thesis]. London: University of London, 1970.

26 Jurvelin J, Säämänen A-M, Arokoski J, Helminen $H$ J, Kiviranta I. Biomechanical properties of the canine knee articular cartilage as related to matrix proteoglycans and collagen. Eng Med 1988; 4: 157-62.

27 Bader D I, Kempson G E, Egan J, Gilbey W, Barret A. The effects of selective matrix degradation on the short-term compressive properties of adult human articular cartilage. Biochim Biophys Acta 1992; 1116: 147-54.

28 Lyyra T, Jurvelin J, Pitkänen P, Väätäinen U, Kiviranta I. Indentation instrument for the measurement of cartilage Indentation instrument for the measurement of cartilage

29 Kiviranta I, Lyyra T, Väätäinen U, Seuri R, Jaroma H, Tammi M, Jurvelin J. Knee joint articular cartilage shows general softening in patients with chondromalacia of the patella. Trans Orthop Res Soc 1995; 20: 197

30 Fosang A J, Neame P J, Hardingham T E, Murphy G, Hamilton J A. Cleavage of cartilage proteoglycan between G1 and G2 domains by stromelysin. F Biol Chem 1991; 24: 15579-82.

31 Rizkalla G, Reiner A, Bogoch E, Poole R. Studies of the articular cartilage proteoglycan aggrecan in health and osteoarthritis. $\mathcal{f}$ Clin Invest 1992; 12: 2268-77. 